Original Article

\title{
Comparison of the effects of visual feedback training and unstable surface training on static and dynamic balance in patients with stroke
}

\author{
Hyuck-soon Hwang, MSc, PT ${ }^{1)}$, Jin-Hong KIm, MSc, PT ${ }^{1)}$, Bo-RAm Choi, PhD, $\left.\mathrm{PT}^{2}\right)^{*}$ \\ 1) Department of Rehabilitation, Gachon University Gil Medical Center, Republic of Korea \\ 2) Department of Physical Therapy, College of Health and Welfare, Silla University: \\ 315 Euisaengmyung-gwan, 700 Bakyangdae-ro, Sasang-gu, Busan 46958, Republic of Korea
}

\begin{abstract}
Purpose] This study compared the effects of visual feedback training and unstable surface training on the static and dynamic balance of stroke patients. [Subjects and Methods] The study enrolled 20 stroke patients and randomly assigned them to visual feedback training and unstable surface training groups. Both groups performed 30 minutes of conventional exercise therapy twice a week for 4 weeks. In addition, the subjects in the visual feedback training group completed a visual feedback training regimen and the subjects in the unstable surface training group completed training on an unstable surface (30-minute session three times a week for 4 weeks in both groups). Static and dynamic balance parameters were recorded immediately before and after the 4 weeks of training. For data analysis, the paired and independent t-test was used to compare the two groups. [Results] In the visual feedback training group, the sway line at the postural sway of the center of pressure and trace length decreased significantly after training. In both groups, the sway range at the limits of stability in the anteroposterior and mediolateral directions increased significantly after training. [Conclusion] Visual feedback training was better at improving static and dynamic balance than unstable surface training in stroke patients.

Key words: Stroke, Unstable surface training, Visual feedback training
\end{abstract}

(This article was submitted Apr. 17, 2017, and was accepted Jun. 26, 2017)

\section{INTRODUCTION}

During the 12-month period after discharge from hospital, 55\% of stroke patients experience a fall, which is a higher rate than that reported in healthy older individuals (52\%). Moreover, $42 \%$ of stroke patients experience repeated falls ${ }^{1)}$, indicating that this population is at a higher risk of falls relative to healthy older individuals. Stroke patients show obvious gait changes, such as short stride length, slow gait velocity, and they lack strength and control of static and dynamic balance ${ }^{2}$. As the ability to balance decreases with age, preventive exercises are needed to reduce the rate of falls among stroke patients.

To improve disordered postural reactions and standing postural reactions, studies have suggested training methods using visual, auditory, and tactile feedback to achieve symmetrical load bearing ${ }^{3}$. Various exercises involving dynamic rhythm or mechanical devices have also been suggested ${ }^{4}$. Decreasing asymmetric postural alignment and strengthening dynamic stability, in stroke patients are important therapeutic targets ${ }^{5)}$. Postural symmetry and dynamic stability can be improved by feedback training using a force plate, and visual feedback on body weight distribution has been reported to be effective at increasing the symmetry of the standing posture in stroke patients ${ }^{6,7)}$. Previous studies have attempted to identify rehabilitation exercises for stroke patients and develop evaluation methods using balance instruments. However, few studies have compared the effects of different types of exercise. Therefore, this study compared the effects of visual feedback training (VFT) and unstable surface training (UST) on the static and dynamic balance ability of stroke patients.

*Corresponding author. Bo-ram Choi (E-mail: boram@silla.ac.kr)

(C2017 The Society of Physical Therapy Science. Published by IPEC Inc.

(c) $(-)$ This is an open-access article distributed under the terms of the Creative Commons Attribution Non-Commercial No Deriva-

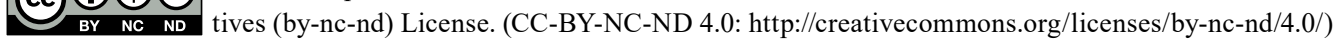




\section{SUBJECTS AND METHODS}

We recruited 20 patients diagnosed with stroke, asked to participate in this study by their physicians, at G University Hospital and G Rehabilitation Hospital (both Incheon, South Korea). After the subjects gave informed consent, they were randomly divided into two groups using table of random numbers: a VFT group (four men, six women; age, $54.30 \pm 7.94$ years; height, $159.00 \pm 7.34 \mathrm{~cm}$; weight, $58.20 \pm 5.24 \mathrm{~kg}$; time since stroke, $14.20 \pm 5.12$ months; affected side, right 6 , left 4 ) and a UST group (three men, seven women; age, $54.80 \pm 13.02$ years; height, $161.30 \pm 4.99 \mathrm{~cm}$, weight $58.80 \pm 8.40 \mathrm{~kg}$; time since stroke, $15.00 \pm 13.02$ month; affected side, right 5, left 5). The inclusion criteria included occurring stroke more than 6 month, being able to walk at least $10 \mathrm{~m}$ with cane or walker, and more than 24 point of Mini-Mental State Examination. The Balance Trainer-4 (BT-4; HUR, Kokkola, Finland) balance assessment device was used to evaluate static and dynamic balance before and after training ${ }^{8)}$. This device has a 100-Hz sampling rate and measuring sensors at each corner of its square platform. The position of the foot was marked on the square platform to assist the subject and the four sensors determined the subject's center of pressure. Balance ability, based on time-dependent postural sway, was measured using Smart-suit Balance software (ver. 1.4; HUR, Kokkola, Finland). Both groups performed conventional exercise therapy for 30 minutes twice a week for 4 weeks. The conventional exercise therapy was designed to correct abnormal posture and movements, and to promote normal exercise patterns to perform various functions and included joint-movement, stretching, and muscle-strengthening exercises $^{9)}$. In addition to this, the VFT group completed a VFT regimen, while the UST group completed a UST regimen. The VFT was done using the BT-4, which includes the Chase, Tennis, and Static Pattern built-in balance-training programs. The patients spent 10 minutes playing each game, giving a 30-minute training session that was done three times a week for 4 weeks. UST, which used a balance pad (Airex, Altenrhein, Switzerland) to provide an unstable surface, was also done for 30 minutes a day, three times a week for 4 weeks. All exercises were administered by a physiotherapist with more than 3 years' worth of treatment experience. The statistical analysis was performed using SPSS for Windows software (ver. 12.0; SPSS Inc., Chicago, IL, USA). The paired t-test was used to compare the results before and after training in each group. The independent t-test was used to compare results between the two groups. The level of statistical significance level $(\alpha)$ was set at 0.05 .

\section{RESULTS}

In the VFT group, the sway line at the postural sway of the center of pressure and the trace length decreased significantly after training, while there was no significant difference in the UST group. The VFT group showed a significantly greater difference before versus after training than did the UST group (Table 1). In both groups, the sway range at the limit of stability in the anteroposterior and mediolateral directions increased significantly after training, and the difference before versus after training was significantly greater in the VFT group than it was in the UST group (Table 2).

Table 1. A comparison of two groups in sway line on postural sway of center of pressure of area and trace length

\begin{tabular}{llllll}
\hline & & Pre-test & Post-test & p \\
\hline \multirow{2}{*}{ Area $\left(\mathrm{mm}^{2}\right)$} & VFT & $436.2 \pm 294.9$ & $401.6 \pm 284.8$ & $*$ \\
& UST & $428.2 \pm 142.1$ & $414.0 \pm 129.2$ & $*$ & $*$ \\
\hline \multirow{2}{*}{ Trace length $(\mathrm{mm})$} & Post -Pre & $-34.6 \pm 19.7$ & $-14.2 \pm 21.3$ & $614.9 \pm 222.7$ & $*$ \\
& VFT & $647.6 \pm 222.7$ & $577.8 \pm 201.9$ & & $*$ \\
\hline
\end{tabular}

VFT: Visual feedback training; UST: Unstable surface training. ${ }^{*} \mathrm{p}<0.05$

Table 2. A comparison of two groups in sway range in limit of stability of antero-posterior and medio-lateral direction

\begin{tabular}{lllll}
\hline & & Pre-test & Post-test & p \\
\hline \multirow{3}{*}{ A-P LOS (mm) } & VFT & $6.33 \pm 1.31$ & $6.84 \pm 1.37$ & $*$ \\
& UST & $6.09 \pm 1.55$ & $6.14 \pm 1.56$ & $*$ \\
& Post -Pre & $0.50 \pm 0.21$ & $0.05 \pm 0.06$ & $*$ \\
\hline \multirow{2}{*}{ M-L LOS (mm) } & VFT & $6.49 \pm 1.18$ & $6.06 \pm 1.29$ & $*$ \\
& UST & $6.94 \pm 1.08$ & $6.18 \pm 1.18$ & $*$ \\
\hline
\end{tabular}

VFT: Visual feedback training; UST: Unstable surface training; A-P LOS: Antero-Posterior limit of stability; M-L LOS:

Medio-Lateral limit of stability. ${ }^{*} \mathrm{p}<0.05$ 


\section{DISCUSSION}

VFT improve both static and dynamic balance and was more effective at improving static balance than UST. Few studies have compared the effect of VFT with other forms of exercise. One study reported that a virtual reality task using visual feedback exercises improved static balance, while there was no difference between visual feedback and unstable surface exercise groups $^{3}$. By contrast, we found that VFT enhanced static balance more than UST. The training might have been more effective in our study because the time since stroke of subjects in our study were less 10 months than subjects in previous study. Furthermore, enhanced dynamic balance was seen in our study. Cheng et al. showed that rhythmic postural change training using visual feedback improved the dynamic balance of patients with hemiplegia; furthermore, the effect of the training persisted for 6 months, and the patients had a reduced risk of falls ${ }^{10}$ ). In a study on weight distribution across both legs during standing, Sackley et al. found that patients with stroke showed recovery of dynamic balance due to an increase in dynamic movement, in turn due to a shift of body weight to the affected side ${ }^{7}$. Limitation of this study was short-term exercise. Future researchers should examine effect of the long-tern exercise. VFT might be effective for preventing falls in patients with stroke because this training improves dynamic and static balance to a greater extent than UST.

\section{REFERENCES}

1) Ashburn A, Hyndman D, Pickering R, et al.: Predicting people with stroke at risk of falls. Age Ageing, 2008, 37: 270-276. [Medline] [CrossRef]

2) Ikai T, Kamikubo T, Takehara I, et al.: Dynamic postural control in patients with hemiparesis. Am J Phys Med Rehabil, 2003, 82: 463-469, quiz 470-472, 484. [Medline] [CrossRef]

3) Sackley C, Baguley B: Visual feedback after stroke with the balance performance monitor: two single-case studies. Clin Rehabil, 1993, 7: 189-195. [CrossRef]

4) Matjacić Z, Rusjan S, Stanonik I, et al.: Methods for dynamic balance training during standing and stepping. Artif Organs, 2005, 29: 462-466. [Medline] [CrossRef]

5) Nichols DS: Balance retraining after stroke using force platform biofeedback. Phys Ther, 1997, 77: 553-558. [Medline] [CrossRef]

6) Liston RA, Brouwer BJ: Reliability and validity of measures obtained from stroke patients using the Balance Master. Arch Phys Med Rehabil, 1996, 77: 425-430. [Medline] [CrossRef]

7) Sackley C, Baguley B, Gent S, et al.: The use of a balance performance monitor in the treatment of weight-bearing and weight-transference problems after stroke. Physiotherapy, 1992, 78: 907-913. [CrossRef]

8) Piirainen JM, Avela J, Sippola N, et al.: Age dependency of neuromuscular function and dynamic balance control. Eur J Sport Sci, 2010, 10: 69-79. [CrossRef] Gjelsvik BEB: The Bobath concept in adult neurology. 2008.

10) Cheng PT, Wang CM, Chung CY, et al.: Effects of visual feedback rhythmic weight-shift training on hemiplegic stroke patients. Clin Rehabil, 2004, 18: 747-753. [Medline] [CrossRef] 